\title{
RETRACTED ARTICLE: A completeness theorem for continuous predicate modal logic
}

\author{
Stefano Baratella ${ }^{1}$
}

Received: 17 March 2014 / Accepted: 24 April 2017 / Published online: 4 May 2017

(C) Springer-Verlag Berlin Heidelberg 2017

The Editor-in-Chief is retracting this article because it was published in error before undergoing peer review. The author agrees with this retraction and will be resubmitting his manuscript for review. The Editor-in-Chief apologizes to the author and to readers. The online version of this article contains the full text of the retracted article as electronic supplementary material.

Electronic supplementary material The online version of this article (doi:10.1007/s00153-017-0554-7) contains supplementary material, which is available to authorized users.

$\otimes \quad$ Stefano Baratella

stefano.baratella@unitn.it

1 Dipartimento di Matematica, Università di Trento, Via Sommarive 14, 38123 Povo, TN, Italy 